\title{
New Technologies in Improving Chemical Properties and Sensory Attributes of
} Olive Oil

\author{
Susan M. M. Abd-Elmageed, Rania I. M. Almoselhy and Badawy H. A. A. \\ Oils and Fats Research Dept., Food Technology Research Institute, Agricultural Research Center, \\ Giza, Egypt
}

Received: 12 August 2019/Accepted 20 Oct. 2019/Publication date: 30 Oct. 2019

\begin{abstract}
This work aimed to improve the quality of lampante virgin olive oil (LVOO) (olive oil with severe defects greater than 6.0 or free acidity greater than 3.3\%). It is not fit for human consumption. LVOO may come from bad fruit from improper handling. Lampante virgin olive oil (LVOO) was mixed with good olive oil cake (OOC) (olive pomace) and good olive mill wastewater (OMWW) by ratio 20: 30: 50. This mixture was transported to malaxer unit, for malaxing at 30, 45, and 60 min. After each malaxation time the paste was sent to the decanter to re-extract the oil. In decanter unit paste was divided into three phases, after decantation the husk that was given out from the machine was transported to the outside of the mill. For recovering the oil, the machine used the centrifugal force (separator), oil and OMWW were transported to separator unit for separation oil from water. During the re-extraction of olive oil with malaxation time 30,45, and $60 \mathrm{~min}$, changes in chemical properties and sensory attributes were studied. Results indicated that sensory attributes were improved at three malaxation time 30, 45, and 60 min, mainly fruitiness and bitterness, the high malaxing time $60 \mathrm{~min}$ was the best. Also results revealed that chemical properties were improved at three malaxation time, mainly free acidity and peroxide value which were decreased, and high malaxation time was the best, olive oil obtained by this techniques may classified as ordinary olive oil (free acidity less than $3.3 \%$ and defect less than 6.0. These results suggested the use of these techniques to improve the quality of LVOO.
\end{abstract}

Keywords: Lampante virgin olive oil (LVOO), new technology, improved olive oil (IOO), sensory quality.

\section{Introduction}

Virgin olive oil is classified in the following manner according to the International Olive Council (IOC) 2018.

1- Extra virgin olive oil when the median of the defects is equal to 0.0 , the median of the fruity attribute is more than 0.0 and free acidity of less than $0.8 \%$.

2- Virgin olive oil when the median of the defects is more than 0.0 and less than or equal to 3.5 , the median of the fruity attribute is more than 0.0 and free acidity of less than $2 \%$.

3- Ordinary virgin olive oil when the median of the defects is more than 3.5 and less than or equal to 6.0 , the median of the fruity attribute is equal to 0.0 and free acidity of less than $3.3 \%$.

4- Lampante virgin olive oil when the median of the defects is more than 6.0 and free acidity greater than $3.3 \%$. It is not fit for human consumption.

The quality of olive oil entering the US food supply is graded by the US Department of Agriculture (USDA). US Extra Virgin Olive Oil "has excellent flavor and odor" and a low free fatty acid content. US virgin Olive Oil "has reasonably good flavor and odor" and a higher free fatty acid content than Extra Virgin Olive Oil. The flavor and aroma of olive oils are determined by sensory evaluation (taste-testing) by trained individuals, whereas the free fatty acid content is measured analytically.

Virgin olive oil is produced by crushing fresh olives, followed by mechanical extraction of the oil. No heat or chemicals are used in this process. For the best quality of olive oil, the fruit must be of high quality. Terms used to refer to the processing of olives for virgin olive oil include first-press, cold-press, or cold-extracted. This type of olive oil is the most flavorful and is potentially the most 
health-enhancing because it contains naturally occurring substances such as polyphenols (Buckland and Gonzalez, 2015).

Giovacchino et al. (1996) studied the effect of mixing leaves with olives on organoleptic quality of oil obtained by centrifugation. The results indicated that variable quantities of leaves were added to ripe olives prior to extraction to determine their effect on the quality of the resulting olive oil. The addition of $1-3 \% \mathrm{w} / \mathrm{w}$ leaves improved the organoleptic quality. An increase in trans-2hexenal aldehyde content occurred, giving the pleasant flavor of freshly cut grass.

Al-Okaby et al. (2015) found that addition aqueous citric acid solution (30\%), as a new technological, to olive paste at different levels $(0.5,1.0,1.5$ and $2.0 \% \mathrm{v} / \mathrm{w})$ during malaxation step improved the yield, total polyphenols, chemical and organoleptic characteristics, oxidative stability and fatty acid composition of the olive oil (Olea europaea L.)

Sensory quality plays an important factor in olive oil overall quality. Aroma, taste and appearance are particularly significant for extra virgin olive oil. Flavor, olive oil quality and hence its commercial value varies markedly depending on several factors. The complex flavor of virgin olive oil is mainly produced by volatile and phenol compounds most of which have been identified (Morales et al., 1997). The quantities of these substances in olive depend on agronomic factors, olive ripeness, cultivar, olive storage conditions, handling, milling, malaxation and extraction processing (by pressing or centrifugation) (Tzia et al., 1997).

In the olive fruits there is a large amount of bioactive compounds and substances of high interest. Many of them are known by owing health beneficial properties that contribute to protective effect of the virgin olive oil. During olive oil processing most of them remain in the olive oil wastes (Juan et al., 2006).

The quality of olive oil could be ever more improved by regulating the malaxing parameters and monitoring the chemical / biochemical changes of olive paste precisely (Jimenez et al., 2005).

The aim of the malaxation is to break up of the oil/ water emulsion and to assure the aggregation of the dispersed oil droplets in the paste to facilitate the subsequent process of the oil separation. In recent years the role of the operative conditions applied during malaxation that largely effect on the concentration of the phenolic and volatile compounds. (Servili et al., 2008).

The beneficial health effects of olive oil are due to both its high content of monounsaturated fatty acids and its high content of antioxidative substances (Visioli and Galli, 1998). The olive oil industry is very important in Mediterranean countries, both in terms of wealth and tradition. In particular, during extraction, the content of some components is significantly modified, depending on technique employed (Morales and Aparicio, 1999).

Sanchez et al. (2003) noted that monounsaturated oleic acid in the formation of a cell membrane which is more resistant to oxidation by acting at the level of greater stability in the cell membrane.

The present work focuses on the development of new technologies by using natural treatment to improve the characteristics of lampante virgin olive oil (LVOO) to be fit for human consumption.

\section{Materials and Methods}

\section{Materials:}

1- The study was carried out in A.B.C. Olive Oil Mill Agriculture Factory Industrial Zone, Wady El-Natron city, Egypt.

2- Lampante virgin olive oil was obtained from factory in El-Khatatba area.

3- Good OOC (by-product) obtained from A.B.C. Olive Oil Mill Agriculture Factory directly after olive oil extraction.

4- Good OMWW (by-product) obtained from A.B.C. Olive Oil Mill Agriculture Factory.

5- All chemical, reagents and solvents used in this study were of analytical grade and obtained from Sigma agent and Cornel Lab companies in Egypt. 
Chemical and organoleptic characteristics of virgin olive oil categories as defined by the IOC 2018.

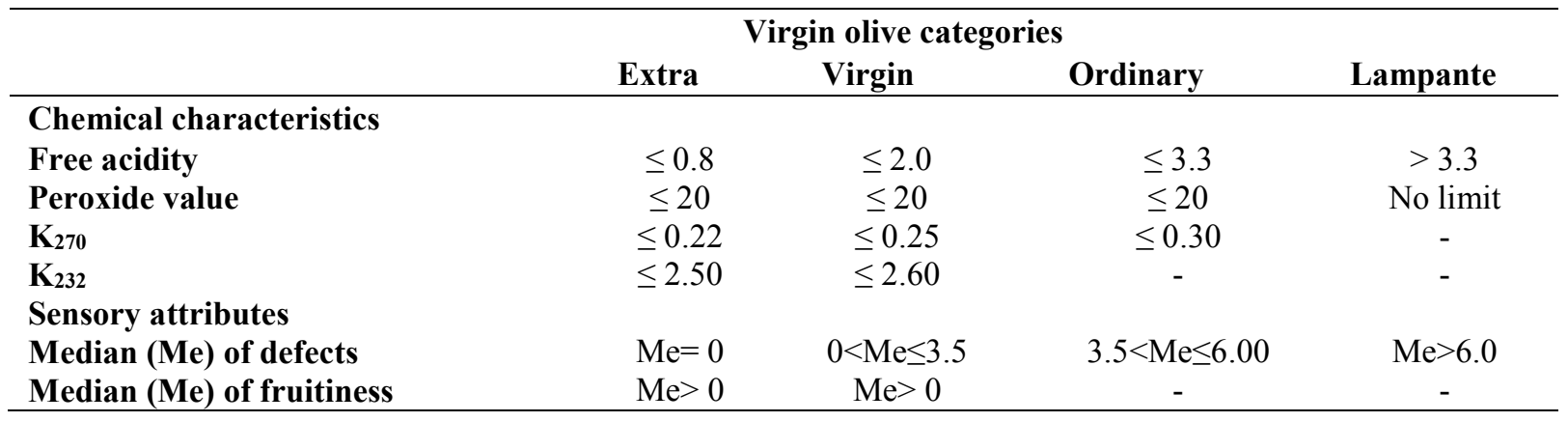

The free acidity is expressed as \% of oleic acid (mass/mass). The peroxide value is given in meq $\mathrm{O}_{2} / \mathrm{kg}$ of olive oil. For other parameters, see explanations in the corresponding text.

The present work focuses on the development of new technologies by using natural treatment to improve the characteristics of lampante virgin olive oil (LVOO) to be fit for human consumption.

\section{Analytical methods:}

\section{Experimental design}

Experimental design was carried out as the following:

Lampante virgin olive oil (LVOO) was mixed with good olive oil cake (OOC) (olive pomace) and good olive mill wastewater (OMWW) by ratio 20: 30: 50. This mixture was transported to malaxer unit.

\section{Scheme of re-extraction line to improve chemical and organoleptic quality of LVOO}
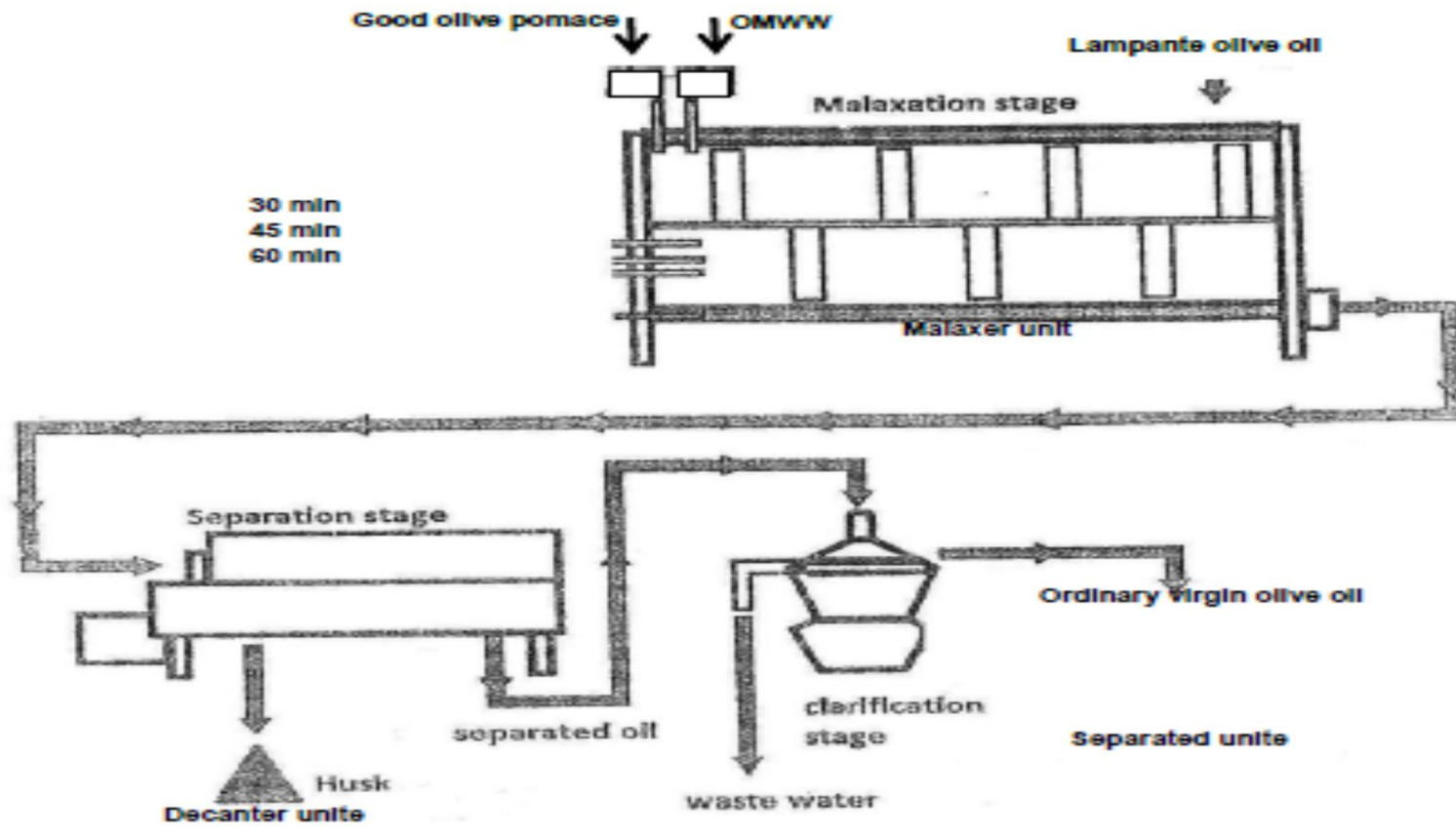

\section{Quality indices}

The chemical indices were analyzed using official methods and the sensory evaluation was performed by Egyptian panelists from Food Technology Research Institute which were officially recognized by IOC (2005) and using IOC sensory evaluation method. 
Free fatty acids (FFA) \% and Peroxide value (PV) meq $\mathrm{O}_{2} / \mathrm{kg}$ oil were evaluated according to the European Union Commission (1991) Regulation EEC/2568/91.

Ultra Violet (UV) absorption was carried out according to IOOC Regulation COI/T20/Dos.No.19/rev. 1 (IOOC 2001). Using a UV spectrophotometer, k232 and K270 were measured, by the absorption at 232 and $270 \mathrm{~nm}$, respectively, in a solution of oil in cyclohexane.

\section{Oxidative stability (OST):}

The induction time (oxidative stability) was evaluated by the Rancimat method (Gutierrez, 1989). Stability was expressed as the oxidation induction time (hr.), measured with the Rancimat 743 apparatus (Metrohm, Basel, Switzerland), using an oil sample of $3 \mathrm{~g}$ heated to $100^{\circ} \mathrm{C}$ and an air flow of $20 \mathrm{~L} \mathrm{hr}^{-1}$.

\section{Fatty acids composition:}

The methyl-esters of fatty acids were prepared from LVOO and obtained improved olive oil (IOO) and after cold saponification by vigorous shaking of a solution of oil in hexane $(0.2 \mathrm{~g}$ in $3 \mathrm{ml})$ with $0.4 \mathrm{ml}$ of $2 \mathrm{~N}$ methanolic potassium hydroxide (IOOC, 2001).

Total phenolic (TP) content: was determined following the procedure indicated by Montedoro et al. (1992).

Identification and determination of phenolic fractions of LVOO and IOO using HPLC: phenolic fraction compounds were identified and determined by the method described by Schieber et al., (2001).

\section{Oil extracted from olive cake:}

Oil was extracted from olive cake using centrifuge method at $6000 \mathrm{rpm}$ for $10 \mathrm{~min}$. The extracted oil was collected to study the characteristics of olive oil cake.

\section{Determination of olive oil extraction yield:}

Olive oil extraction yield was calculated taking into account the flow rate of the olive paste $(\mathrm{kg} / \mathrm{h})$, the production time $(\mathrm{h})$ and the final olive oil recovered after natural decantation $(\mathrm{kg})$. The extraction yield was expressed in $\mathrm{kg}$ oil/100 kg of olive paste Puertolas and Martinez (2015).

\section{Sensory analysis:}

A sensory analysis (median of defects, median of fruity and panel classification test) of the samples was carried out by 8 trained panelists, according to the method described in Regulation EEC 640/2008 (European Union Commission, 2008). The intensities of both positive (fruity, bitter and pungent) and negative (fusty, winy, musty, muddy, rancid, metallic, and other) attributes were evaluated for each oil sample, on a non-structured, $10 \mathrm{~cm}$ scale. The different attributes of the oils were assessed and their intensities were evaluated as a median value of the panelists score.

\section{Results and Discussion}

Lampante virgin olive oil (LVOO) was mixed with olive pomace resulted from previously extracted olive oil with good olive oil characteristics. Oil was extracted from olive cake by centrifuge method and its free fatty acids, peroxide value, K232, K270, total phenols and induction time were determined and the results are tabulated in Table (1). The content of free acidity and peroxide value that we have recorded in olive cake oils values below the limit value, $0.8 \%$ and $20 \mathrm{meq} / \mathrm{kg}$ respectively, recommended by IOC (2018) for extra virgin olive oils. For the other parameters K232 and $\mathrm{K} 270$, the value obtained in the limits recommended by the IOC for virgin olive oils. Total phenols $\mathrm{mg} / \mathrm{L}$ and induction time (h) at $100^{\circ} \mathrm{c}$ were to be $195.15 \mathrm{mg} / \mathrm{L}$ and $13.8 \mathrm{~h}$.

\section{Influence of the new technology on characteristics of olive oil produced from mixing LVOO, OOC and OMWW:}

The influence of mixing LVOO with OOC and OMWW (20:30:50) at different malaxation time (30, 45 and $60 \mathrm{~min})$ on the quality of LVOO was determined and results are tabulated in Table 
(1). Results revealed that there was an increase in the oil extraction yield with the increasing in malaxation time. The application of this technique resulting in decreasing the free acidity of oil sample from 4.8 to $3.82,3.11$, and $2.84 \%$ for IOO after 30,45 , and 60 min of malaxation time, respectively. Peroxide value measured the hydroperoxides formed during the primary stage of oxidation of unsaturated fatty acids of the triglycerides; peroxide value is used as an indicator for revealing defects related to enzymatic and oxidative deterioration in oils. Results in Table (1) showed that the qualitative parameters of olive oil, like peroxide value, spectrophotometric absorption in the UV (K232 and K270) were decreased with the increment of the malaxation time. The absorbency at $232 \mathrm{~nm}$ is used as an indication of hydroperoxides formed in the primary stage of oxidation and conjugated dines formed in the intermediate stage of oxidation. The absorbency at $270 \mathrm{~nm}$ is measured the carbonylic compounds (ketones and aldehydes) formed in the secondary stage of oxidation. These results indicated that oil sample after $45 \mathrm{~min}$ and $60 \mathrm{~min}$ of malaxation produced an olive oil could be classified as ordinary virgin olive oil according to EU legislation (European Union Commission (2003) Regulation EC 1989/2003) and IOC (2018). In the same time total phenol content was increased with the increment in the malaxation time from $126 \mathrm{mg} / \mathrm{L}$ for LVOO to 132.26, 149.23, and $162.56 \mathrm{mg} / \mathrm{L}$ for the oil obtained after malaxation time 30, 45, and $60 \mathrm{~min}$, respectively. Also induction time (h) increased from $7.1 \mathrm{~h}$ for LVOO to $9.13 \mathrm{~h}$ for IOO after $60 \mathrm{~min}$ of malaxation time.

Table 1: Characteristics of olive oil produced from mixing LVOO, OOC and OMWW at different malaxation time

\begin{tabular}{|c|c|c|c|c|c|}
\hline \multirow[b]{2}{*}{$\begin{array}{l}\text { Chemical } \\
\text { characteristics }\end{array}$} & \multirow{2}{*}{ LVOO } & \multirow{2}{*}{$\begin{array}{l}\text { Oil obtained } \\
\text { from olive } \\
\text { cake }\end{array}$} & \multicolumn{3}{|c|}{$\begin{array}{c}\text { Oils obtained after treatments } \\
\text { Malaxation time }\end{array}$} \\
\hline & & & $30 \mathrm{~min}$ & $45 \mathrm{~min}$ & $60 \mathrm{~min}$ \\
\hline Free fatty acids (\%) & 4.80 & 0.25 & 3.82 & 3.11 & 2.84 \\
\hline Peroxide value (meq $\mathrm{O}_{2} / \mathrm{kg}$ oil) & 26.15 & 3.20 & 18.43 & 16.55 & 14.86 \\
\hline $\mathbf{K}_{232}$ & 3.22 & 2.15 & 2.86 & 2.53 & 2.15 \\
\hline $\mathbf{K}_{270}$ & 0.60 & 0.26 & 0.41 & 0.38 & 0.31 \\
\hline Total phenols (mg/L) & 126.00 & 195.15 & 132.26 & 149.23 & 162.56 \\
\hline Induction time (h) at $100^{\circ} \mathrm{c}$ & 7.10 & 13.80 & 7.74 & 8.91 & 9.13 \\
\hline Yield (kg oil/ 100kg olive paste) & - & - & 20.20 & 20.41 & 20.58 \\
\hline
\end{tabular}

*Oils obtained after mixing with OOC and OMWW at different malaxation time

\section{Fatty acids composition}

Fatty acids profile presented in Table (2) for LVOO before and after treatment (mixing with OOC and OMWW and malaxed for $60 \mathrm{~min})$ showed that, their main fatty acids were Oleic (73.14 and $74.79 \%$ ) and Palmitoleic (1.53 and 1.24\%); the monounsaturated fatty acids, Palmitic (8.52 and $8.13 \%)$ and Stearic (3.14 and 3.32\%); the saturated fatty acids, Linoleic (12.00 and $10.82 \%)$ and Linolenic (1.00 and $0.90 \%$ ); the polyunsaturated fatty acids. Other fatty acids presented at low concentration was Arachidic $(0.30$ and $0.38 \%)$, respectively. Olive oil is classified as a monounsaturated fat because of the predominance of Oleic acid.

Results in Table (2) shows that, the predominant fatty acids in LVOO and IOO were six primary fatty acids including oleic, linoleic, palmitic and stearic which are the main ones representing about $97.17 \%$ and $97.48 \%$ of the composition of total fatty acids of both LVOO and IOO, respectively. Furthermore palmitoleic, linolenic and arachidic acids were found in small quantities; $2.83 \%$ and $2.52 \%$ in LVOO and IOO, respectively. These results are in agreement with that reported by Fuentes et al. (2015), how reported that the main fatty acids are oleic, linoleic, palmitic and stearic which are representing $99 \%$ of the composition of total fatty acids in virgin olive oil.

Also, results revealed that the IOO contained higher amount of oleic acids and total monounsaturated fatty acids 74.67 and $76.03 \%$, respectively, while polyunsaturated fatty acid was lower in IOO $11.72 \%$ than LVOO $13.00 \%$. The total saturated/ total unsaturated fatty acid ratio was the same $0.14 \%$ in both oils while the monounsaturated /polyunsaturated fatty acids ratio was higher in IOO. The major saturated fatty acid was palmitic acid while oleic acid was predominant unsaturated fatty acid followed by linoleic acid, and the least unsaturated fatty acid percentage was the linolenic acid. 
This means that IOO contains more monounsaturated fatty acids, same amount of total unsaturated fatty acids and less amount of polyunsaturated fatty acids.

Table 2: Fatty acids composition (\%) of LVOO and IOO

\begin{tabular}{lcc}
\hline Fatty acids & LVOO & Olive oil after treatment* \\
\hline Palmitic C16:0 & 8.89 & 8.55 \\
Palmitoleic C16:1 & 1.53 & 1.24 \\
Stearic C18:0 & 3.14 & 3.32 \\
Oleic C18:1 & 73.14 & 74.79 \\
Linoleic C18:2 & 12.00 & 10.82 \\
Linolenic C18:3 & 1.00 & 0.90 \\
Arachidic C20:0 & 0.30 & 0.38 \\
Total saturated fatty acid & 12.33 & 12.25 \\
Total unsaturated fatty acid & 87.67 & 87.75 \\
Monounsaturated fatty acid & 74.67 & 76.03 \\
Polyunsaturated fatty acid & 13.00 & 11.72 \\
Total saturated/total unsaturated fatty acid & 0.14 & 0.14 \\
Monounsaturated/ Polyunsaturated fatty acid & 5.74 & 6.48 \\
\hline
\end{tabular}

*Olive oil obtained after mixing with OOC and OMWW and malaxing for $60 \mathrm{~min}$

\section{Phenolic compounds:}

The olive fruits contain a wide variety of phenolic compounds (Bianchi, 2003 and Owen, 2003). They are potent antioxidants and play an important role in the chemical, organoleptic and nutritional properties of the virgin olive oil.

The amount of phenolic compounds in virgin olive oil is an important factor when we assess their quality, due to the fact that natural phenolic compounds are able to improve the oxidation resistance and because these components are beneficial to human health.

Several phenolic compounds were presented in both olive oil samples (LVOO and IOO) Table (3), however only thirteen compounds were identified. Pyrogallol, tyrosol, catechin, chlorogenic and oleuropein were the predominant phenolic compounds in LVOO and IOO. Also results indicated that increased malaxation time resulted in an increased amount of phenolic compounds content it may be due to an enhanced release of oil constituents from olive tissue (Ranalli et al., 2001). When malaxation time were increased it led to an increase of phenolic compound concentration. Such a behavior is in good agreement with a previous work (Srvilli et al., 2003).

Table 3: Phenolic compounds of LVOO and improved olive oil

\begin{tabular}{lcccc}
\hline Phenolic compounds & LVOO & Oils obtained after treatment* \\
Malaxation time & \\
& & $\mathbf{3 0}$ min & $\mathbf{4 5}$ min & $\mathbf{6 0}$ min \\
\hline Benzoic & 138.53 & 166.36 & 178.05 & 209.17 \\
Caffeine & 202.56 & 226.32 & 252.13 & 290.05 \\
Caffeic & 28.34 & 28.25 & 30.15 & 34.18 \\
Chlorogenic & 211.82 & 250.60 & 289.19 & 395.66 \\
Vanillic & 184.00 & 201.00 & 243.11 & 233.65 \\
Pyrogallol & 165.94 & 180.45 & 205.03 & 236.02 \\
Catechein & 149.45 & 149.00 & 180.00 & 231.32 \\
Ferulic & 87.15 & 87.10 & 88.99 & 92.06 \\
Tyrosol & 150.87 & 181.65 & 199.15 & 272.44 \\
Oleuropein & 134.27 & 159.27 & 177.50 & 267.44 \\
Coumarin & 95.66 & 103.08 & 11.22 & 124.58 \\
p-Coumaric & 35.82 & 35.99 & 39.63 & 41.63 \\
Gallic & 92.63 & 105.11 & 109.85 & 120.15 \\
Unknown & 87.18 & 88.71 & 91.15 & 115.24 \\
Unknown & 5.69 & 5.05 & 6.99 & 7.80 \\
\hline
\end{tabular}

*Oils obtained after mixing with OOC and OMWW at different malaxation time. 
The main phenolic compounds presented in LVOO, and IOO (oil obtained after mixing technique and malaxed for 30,45, and $60 \mathrm{~min}$ are chlorogenic 211.82, 250.60, 289.19, and 395.66 followed by caffeine 202.56, 226.32, 252.13, and 290.05; vanillic 184.00, 201.00, 243.11, and 233.65 and pyrogallol 165.94, 180.45, 205.03, and 236.02, respectively.

These results are in agreement with that reported by Giovacchino et al., (2002), who mentioned that phenolic compounds increased with the increment in malaxation time.

\section{Organoleptic assessment}

Table (4) shows the intensity attributes of LVOO and IOO (during 3 different malaxation time) perceived by eight testers. Both LVOO and IOO showed sensory profiles belonging to olive oil category (Regulation EC 640/2008). From a practical point of view, this new technology improved sensory properties. An important result was the decrease of negative attributes (defects) from $(\mathrm{Me}>6.0)$ to $(\mathrm{Me}<2.5)$ after 60 min of malaxation. The fruity, bitter and pungent attributes showed an increase trend with the increment of malaxation time. The median of the defect was $>6.0$ and the median of the positive (fruity attributes) $>0.00$ for LVOO. Moreover, samples after used this new technology improved the median of defects was $(2.5<$ me $<6)$ after 30 min malaxation. Also results show slightly increase of the median of positive attributes, close to the same results for samples after 45 min malaxation while the median of defects was $(2.5<$ me $<6)$. Sample after 60 min malaxation may classified as ordinary virgin olive oil based on sensory analysis and limits of IOC standard (2018) (median of defects $3.5 \geq \mathrm{me}<6$ and fruity $=0.00$ )

Table 4: Organoleptic assessment of LVOO before and after treatment

\begin{tabular}{|c|c|c|c|c|}
\hline \multicolumn{2}{|c|}{ Samples } & \multicolumn{3}{|c|}{$\begin{array}{l}\text { Oils obtained after treatment * } \\
\text { Malaxation time }\end{array}$} \\
\hline $\begin{array}{l}\text { Sensory } \\
\text { Attributes }\end{array}$ & LVOO & $30 \mathrm{~min}$ & $45 \mathrm{~min}$ & $60 \mathrm{~min}$ \\
\hline Median (Me) of fruity & 1.3 & 1.8 & 2.2 & 2.7 \\
\hline Median (Me) of bitter & 0.8 & 1.1 & 2.1 & 2.4 \\
\hline Median (Me) of pungent & 1.2 & 1.9 & 2.1 & 2.5 \\
\hline Median (Me) of defect & $\mathrm{Me}>6.0$ & $2.5<\mathrm{Me}<6.0$ & $\mathrm{Me}<6.0$ & $0.0<\mathrm{Me}<2.5$ \\
\hline
\end{tabular}

*Oils obtained after mixing with OOC and OMWW at different malaxation time

\section{Practical applications:}

The results obtained clearly open new lines of research to use fresh olive cake and OMWW a by-product of olive oil extraction as natural sources to improve the chemical and organoleptic quality of low quality olive oil.

\section{Conclusion}

It was concluded that, mixing $20 \%$ of LVOO with $30 \%$ good OOC and $50 \%$ good OMWW has improved both chemical properties and sensory attributes of LVOO and this was more pronounced with the increased in malaxation time during re-extraction of olive oil. This new and naturally technology can be used to enhance low quality olive oil not suitable for consumption and produce good characteristics olive oil fit for consumption.

\section{References}

Al-Okaby, M. F., M.Rasmy Nagwa, M. H. El-Kalyoubi, and A.Y. Girgis, 2015. Improving the extraction efficiency and quality of virgin olive oil using citric acid. Middle East Journal of Applied Sciences, 05: 148-156.

Bianchi, G., 2003. Lipids and phenols in table olives. Eur. J. Lipid Sci. Technol., 105: 229-242.

Buckland, G., and C.A. Gonzalez, 2015: The role of olive oil in disease prevention: a focus on the recent epidemiological evidence from cohort studies and dietary intervention trials. Br. J. Nutr. 113 Suppl. 2: S94: 101.

European Union Commission, 1991. Regulation (ECC) No 2568/91. Official Journal of the European Communities, L248. 
European Union Commission, 2003. Regulation (EC) No 1989/2003. Official Journal of the European Communities, L295.

European Union Commission, 2008. Characteristic of olive oil and olive-residue oil and on the relevant methods of analysis Regulation EEC/640/2008. O. J. Eur. Comm. L178, 11-16.

Fuentes, M., C. D. Miguel, A. Ranalli, M. N. Franco, M. Martinez, and D. Martin-Vertedor, 2015: Chemical composition and sensory evaluation of virgin olive oils from Morisca and Carrasquena olive varieties. Grasas Y Aceites, 66 (1):1-16

Giovacchino, L. D., F. Angerosa, and D. L. Giacinto, 1996. Effect of mixing leaves with olives on organoleptic quality of oil obtained by centrifugation. JAOCS 73:371-374.

Giovacchino, L.D., N. Costantini, M.L. Ferrante and A. Serraiocco, 2002: Influence of malaxation time of olive paste on the technological results and qualitative characteristics of virgin olive oil obtained by a centrifugal decanter at water saving. Grasas Aceites 53, 179-186.

Gutierrez, F., 1989. Determinacion de la estabilidade oxidativa de aceites de oilva virgenes: Comparacion entre del metodo A.O.M. yel metodo Rancimat. Grasas. Aceites, 40: 1-5.

IOOC (International Olive Oil Council), 2001. Preparation of the fatty acid methyl ester from olive oil and olive pomace oil. International Olive Oil Council, Madrid.

IOOC (International Olive Oil Council), 2005. Method IOOC for the organoleptic assessment of virgin olive oil. XV International course on the improvement of olive oil quality (Cairo, Egypt, 20 February- 27 February 2005)

IOC (International Olive Council), 2018.Trade Standard Applying to Olive Oils and Olive Pomace oils. COI/T.15/NC NO 3/ Rev. 12 June 2018

Jimenez Marquez, A., A. Molinaiaz, and M. I. Pascual Reguera, 2005. Using optical NIR sensor for on-line virgin olive oils characterization. Sensors and Actuators B, 107: 64-68.

Juan, F.B., R. Guillermo, R. Rocio, G. Rafael, and J. Ana, 2006. Extraction of interesting organic compounds from olive oil waste. Grasas Y Aceites, 57: 95-106.

Montedoro, G.F., M. Servili, M. Balbioli, and E. Miniati, 1992. Simple and hydrolysable phenolic compounds in virgin olive oil. 2. Initial characterization of the hydrolysable fraction. Journal of Agricultural and Food Chemistry, 40: 1577-1580.

Morales, M. T., J. J. Rios, and R. Aparicio, 1997: Changes in the volatile composition of virgin olive oil during oxidation: Flavors and off-flavors. J. Agric. Food Chem., 45: 2666-2673.

Morales, M. T. and R. Aparicio, 1999: Effect of extractions on sensory quality of virgin olive oil. JAOCS, 76: 295-300.

Owen, R., R. Haubner, W. Mier, A. Giacosa, W. Hull, B. Spiegelhalder, and H. Bartsch, 2003. Isolation, structure, elucidation and antioxidant potential of the major phenolic and flavonoid compounds in brined olive drupes. Food and Chemical Toxicology, 41: 703-717.

Puertolas, E. and I. M. Martinez, 2015. Olive oil pilot-production assisted by pulsed electric field: Impact on extraction yield, chemical parameters and sensory properties. Food Chemistry, 167: 497-502.

Ranalli, A., S. Contento, C. Schiavone and N. Simone, 2001. Malaxing temperature affects volatile and phenol composition as well as other analytical features of virgin olive oil. Eur. J. Lipid Sci. Technol., 103: 228-238

Sanchez, J., E. Osorio, A.M. Montano, and M. Martinez, 2003. Estudio del contenido en acidos grasos de aceites monovarietales elaborados a partir de aceitunas producidas en la region extremena. Grasas Aceites 54, 371-377.

Schieber, A., P. Keller, and R. Carle, 2001. Determination of phenolic acids and flavonoids of apple and pear by high performance liquid chromatography system. J. of Chromatography A, 910: 265-273.

Servili, M., R. Selvaggini, A. Taticchi, S. Esposto, and G. Montedoro, 2003. Air exposure time of olive pastes during the extraction process and phenolic and volatile composition of virgin olive oil. Journal of American Oil Chemists Society, 80: 685-695.

Servili, M., A. Taticchi, S. Esposto, S. Urbani, R. Selvaggini, and G. F. Montedoro, 2008. Influence of the decrease in oxygen during malaxation of olive pastes on the composition of volatiles and phenolic compounds in virgin olive oil. Journal of Agricultural and Food Chemistry, 56: 10048-10055. 
Tzia, C., E. Touzopoulou, and S. Kontou, 1997. Sensory control of olive oil. Acta. Hort., 474: 663666.

US Department of Health and Human Services and U.S. Department of Agriculture. (USDHHS and USDA). 2015. 2015-2020 Dietary Guidelines for Americans. $8^{\text {th }}$ Edition. Available at http://health.gov/dietaryguidelines/2015/guidelines/.

Visioli, F. and C. Galli, 1998. The effect of minor constituents of olive oil on cardiovascular disease: new findings. Nutr. Rev, 56: 142-147. 